\title{
Assessment of contemporary genetic diversity and inter-taxa/inter-region exchange of avian paramyxovirus serotype 1 in wild birds sampled in North America
}

Andrew M. Ramey ${ }^{1 *}$, Iryna V. Goraichuk², Joseph T. Hicks ${ }^{3}$, Kiril M. Dimitrov², Rebecca L. Poulson ${ }^{4}$, David E. Stallknecht ${ }^{4}$, Justin Bahl ${ }^{3}$ and Claudio L. Afonso ${ }^{2}$

\begin{abstract}
Background: Avian paramyxovirus serotype 1 (APMV-1) viruses are globally distributed, infect wild, peridomestic, and domestic birds, and sometimes lead to outbreaks of disease. Thus, the maintenance, evolution, and spread of APMV-1 viruses are relevant to avian health.

Methods: In this study we sequenced the fusion gene from 58 APMV-1 isolates recovered from thirteen species of wild birds sampled throughout the USA during 2007-2014. We analyzed sequence information with previously reported data in order to assess contemporary genetic diversity and inter-taxa/inter-region exchange of APMV-1 in wild birds sampled in North America.

Results: Our results suggest that wild birds maintain previously undescribed genetic diversity of APMV-1; however, such diversity is unlikely to be pathogenic to domestic poultry. Phylogenetic analyses revealed that APMV-1 diversity detected in wild birds of North America has been found in birds belonging to numerous taxonomic host orders and within hosts inhabiting multiple geographic regions suggesting some level of viral exchange. However, our results also provide statistical support for associations between phylogenetic tree topology and host taxonomic order/region of sample origin which supports restricted exchange among taxa and geographical regions of North America for some APMV-1 sub-genotypes.

Conclusions: We identify previously unrecognized genetic diversity of APMV-1 in wild birds in North America which is likely a function of continued viral evolution in reservoir hosts. We did not, however, find support for the emergence or maintenance of APMV-1 strains predicted to be pathogenic to poultry in wild birds of North America outside of the order Suliformes (i.e., cormorants). Furthermore, genetic evidence suggests that ecological drivers or other mechanisms may restrict viral exchange among taxa and regions of North America. Additional and more systematic sampling for APMV-1 in North America would likely provide further inference on viral dynamics for this infectious agent in wild bird populations.
\end{abstract}

Keywords: Avian paramyxovirus serotype 1, APMV-1, Exchange, Genetic diversity, Migratory bird, Newcastle disease, Order, Region, Taxa, Wild bird

\footnotetext{
*Correspondence: aramey@usgs.gov

'US Geological Survey, Alaska Science Center, 4210 University Drive,

Anchorage, Alaska 99508, USA

Full list of author information is available at the end of the article
} 


\section{Background}

Avian paramyxovirus serotype 1 (APMV-1) viruses are globally distributed and infect wild, peridomestic, and domestic birds. Virulent strains of APMV-1 have caused periodic epornitics of Newcastle disease in doublecrested cormorants (Phalacrocorax auritus; order Suliformes) in North America [1-5]; been associated with outbreaks of disease in pet birds and those kept as part of zoological collections [6-8]; and are among the most economically costly poultry pathogens worldwide [9]. APMV-1 strains of low virulence and those considered to be non-pathogenic are also geographically widespread and have been detected in free-ranging populations of wild and peridomestic birds [10-13]; caged birds including animals maintained at zoos [14, 15]; and poultry reared using a wide range of production practices [16-19]. There is evidence to suggest that virulent APMV-1 strains and those of low virulence may be transmitted across wild bird-poultry interfaces [15, 20-23] and that avirulent viruses may develop increased pathogenicity in poultry [24]. Thus, the maintenance, evolution, and spread of APMV-1 viruses are relevant to the health of wild, captive, and domestic bird populations.

Despite the existence of a relatively broad body of literature on the geographic and host distribution of APMV-1 genotypes [25], much less information is available regarding viral exchange among avian species and dispersal across the landscape, particularly for viruses maintained in wild bird reservoirs. In North America, monoclonal antibody typing and sequencing of the fusion cleavage site for APMV-1 isolates derived from double-crested cormorants, a white pelican (Pelecanus erythrorhynchos), and a ring-billed gull (Larus delawarensis) associated with mortality events in Saskatchewan, Ontario, and Minnesota in 1990 and 1992 provided support for interspecies exchange of virulent APMV-1 strains among wild waterbirds and geographic spread within the mid-continent [20]. Likewise, genetically similar virulent APMV-1 strains detected in double-crested cormorants (Maine, Minnesota, Massachusetts, New Hampshire), great black-backed gulls (Larus marinus; Maine), a great cormorant (Phalacrocorax carbo; New Hampshire), and a herring gull (Larus argentatus; Maryland), associated with mortality events in 2010 also supports interspecies exchange and viral dispersal between Midwestern and Eastern regions of the United States of America (USA) [5]. Additionally, lentogenic APMV-1 strains, virtually genetically identical at the fusion gene, were detected among waterfowl and shorebirds sampled in Alaska during 2007 and 2009 providing evidence of viral exchange among these taxa [13]. Therefore, preliminary evidence supports the exchange of APMV-1 strains among wild waterbird taxa and dispersal among regions. However, the extent of viral exchange between wild bird taxa and among regions of North America has not been thoroughly assessed.

In this investigation, we assessed contemporary genetic diversity and evidence for inter-taxa/inter-region exchange of APMV-1 in wild birds sampled in North America. Specific objectives were: (1) to use contemporary APMV-1 isolates to identify previously unrecognized viral genetic diversity in wild birds inhabiting North America, (2) to assess viral exchange among taxonomic orders of wild birds including the potential for maintenance of viral genotypes in specific host taxa, and (3) to evaluate the dispersal of APMV-1 strains among geographic regions within North America. Results of this study provide information on viral dynamics of APMV-1 in migratory birds that may ultimately prove useful towards understanding risk associated with the emergence, exchange, and dispersal of virulent APMV-1 strains within North America.

\section{Methods}

APMV-1 viruses were recovered via isolation in embryonated specific pathogen free eggs inoculated with swab samples collected from wild migratory birds. These isolations were opportunistic as they recovered as part of influenza A research and surveillance conducted throughout the USA during 2007-2014 [26-28]. RNA was extracted from allantoic fluid using the MagMAX AI/NDV RNA extraction kit (Ambion Inc., Austin, TX) for 58 APMV-1 isolates originating from ducks (Anas crecca, A. discors, $A$. platyrhynchos, A. rubripes, A. strepera, Aythya americana), gulls (Larus argentatus, L. delawarensis, L. marinus, Leucophaeus atricilla, Leucophaeus pipixcan), and shorebirds (Arenaria interpres, Calidris canutus) sampled in Delaware $(n=1)$, Louisiana $(n=4)$, Minnesota $(n=32)$, North Dakota $(n=3)$, New Jersey $(\mathrm{n}=8)$, New York $(n=$ $1)$, and Texas $(n=9$; Additional file 1 : Table S1). The complete coding region of the fusion gene was sequenced using previously described protocols [13] and primers [29]. GenBank accession numbers for complete coding sequences for fusion protein genes generated as part of this study are: KX857666-KX857723. Additionally, complete coding nucleotide sequences for fusion genes of all previously reported APMV-1 isolates were downloaded from the National Center for Biotechnology Information's GenBank public database [30] on 26 August $2016(n=1835)$.

To assess contemporary genetic diversity, we applied Maximum Likelihood (ML) phylogenetic analyses to all publically available full fusion gene sequences and those obtained in this study using MEGA6 [31]. Duplicate and recombinant sequences, sequences of man-made clonal viruses, and sequences that were obtained from vaccinelike viruses were removed from the dataset in order to analyze only viruses representing natural circulation and 
evolution in reservoir and spill-over hosts. Preliminary pair-wise nucleotide distances were computed for all remaining fusion gene sequences, including those obtained in the current study, using MEGA6 [31] and viruses were assigned to either class I or class II per conventional classification of APMV-1 isolates. Thus, the final total of 1,483 fusion gene sequences for APMV-1 isolates was parsed into two smaller datasets representing class $\mathrm{I}(n=211)$ and class II $(n=1272)$ sequences (Additional file 1: Table S1).

For sequences of each class I and class II, phylogenies for taxonomic assignment of genotypes/sub-genotypes were reconstructed by first assessing best-fit substitution models using MEGA6, and the goodness-of-fit for each model was measured by corrected Akaike Information Criterion (AICc) and Bayesian Information Criterion (BIC). Class I and class II trees were reconstructed using the Maximum Likelihood method based on Tamura 3parameter and General Time Reversible models, respectively, as implemented in MEGA6 with 1000 bootstrap replicates. Estimates of average evolutionary distances among clades were inferred using the Maximum Composite Likelihood model as implemented in MEGA6 [32]. For all analyses, codon positions included were 1st $+2 \mathrm{nd}+3 \mathrm{rd}+$ Noncoding and all positions containing gaps and missing data were eliminated. Fusion gene sequences were assigned to genotypes and sub-genotypes per criteria put forth by Diel et al. [33]. These criteria included average distance per site $>0.1$ among genotypes and 0.03-0.1 among sub-genotypes, bootstrap support value at nodes defining genotypes/sub-genotypes $>60 \%$, and the isolation of at least four independent viruses without a direct epidemiologic link for both genotypes and sub-genotypes.

To assess inter-taxa and inter-region exchange of APMV-1 in wild birds sampled in North America, we restricted our data to full-length fusion gene sequences for samples originating from free-ranging taxa sampled in the USA and Canada while excluding sequences from hosts which we inferred to be domestic poultry (chicken, duck, goose, turkey, and pheasant), caged or captive birds (parrots), and taxa of unknown domestic/peridomestic/captive status (i.e., hosts identified as 'avian' or 'fowl'). Furthermore, we excluded sequences from environmental samples from unspecified hosts and those for which species and/or location information was incomplete. Finally, sequences obtained from pigeons were also removed from the dataset because of the synanthropic nature of these birds and a lack of information on public databases for distinguishing between wild and domestic show/race birds. Our resulting data set included 180 sequences (Table 1) for the full fusion gene of APMV-1 isolates originating from wild avian hosts of four orders (Anseriformes, Charadriiformes, Pelecaniformes, and Suliformes). Isolates were assigned to six broad geographic
Table 1 Distribution of APMV-1 isolates genetically characterized for this study by host order and geographic region of sample origin

\begin{tabular}{llllll}
\hline Region & Anseriformes & Charadriformes & Pelecaniformes & Suliformes & Total \\
\hline Alaska & 48 & 1 & 0 & 0 & 49 \\
Canada & 0 & 0 & 0 & 3 & 3 \\
East & 10 & 19 & 0 & 9 & 38 \\
Gulf & 14 & 0 & 0 & 3 & 17 \\
Coast & & & & & \\
Midwest & 45 & 2 & 1 & 16 & 64 \\
West & 2 & 0 & 0 & 7 & 9 \\
Total & 119 & 22 & 1 & 38 & 180 \\
\hline
\end{tabular}

regions prior to further analysis: Alaska, Canada, the Western USA (West; California, Idaho, Nevada, Oregon), the Midwestern USA (Midwest; Michigan, Minnesota, North Dakota, Ohio, Wisconsin), the Gulf Coast USA (Gulf Coast; Florida, Louisiana, Texas), and the Eastern USA (East; Connecticut, Delaware, Massachusetts, Maryland, Maine, New Hampshire, New Jersey, New York, Pennsylvania). All 180 sequences were then analyzed to assess for correlations between tree topology and host taxonomic order or sample region.

ML phylogenetic reconstruction of evolutionary history for North American wild bird APMV-1 isolates was conducted to evaluate consistency among ML and Bayesian approaches used throughout this study. The ML phylogenetic tree was re-constructed using Randomized Axelerated Maximum Likelihood (RAxML) v8.0.0 [34] using a general time-reversible model and gamma distributed rate variation among sites. To assess clade support, bootstrapping was performed with bootstrap convergence criterion, yielding 400 bootstrap iterations. Bayesian phylogenetic analysis was conducted using MrBayes v3.2 [35]. Two independent runs of 10 million generations each were performed using the same nucleotide evolution model described above with default priors. Bayesian Tip-association Significance testing (BaTS) [36] was then used to assess the correlation between phylogenetic topology and the character states of host order and geographic location while taking into account the uncertainty produced by phylogenetic error, using the posterior set of trees produced by our Bayesian Markov Chain Monte Carlo analysis and incorporating a $10 \%$ burn-in of each run's chain length. For every tree in this set, BaTS calculated an Association Index (AI) [37], a Fitch parsimony score (PS) [38] and, for each trait, a monophyletic clade size statistic (MC) [36]. A p-value was produced by comparing the posterior distribution of phylogeny-trait statistics to the null distribution generated from 
randomized sets of taxon-character associations selected from the observed data. $P$-values $\leq 0.05$ were used as evidence to support associations between tip traits (i.e., host order and geographic region of origin) and tree topology.

\section{Results}

Assessment of contemporary genetic diversity

ML phylogenetic analyses of fusion gene sequences for APMV-1 class I isolates from wild, peridomestic, and domestic birds and 32 closely related sequences

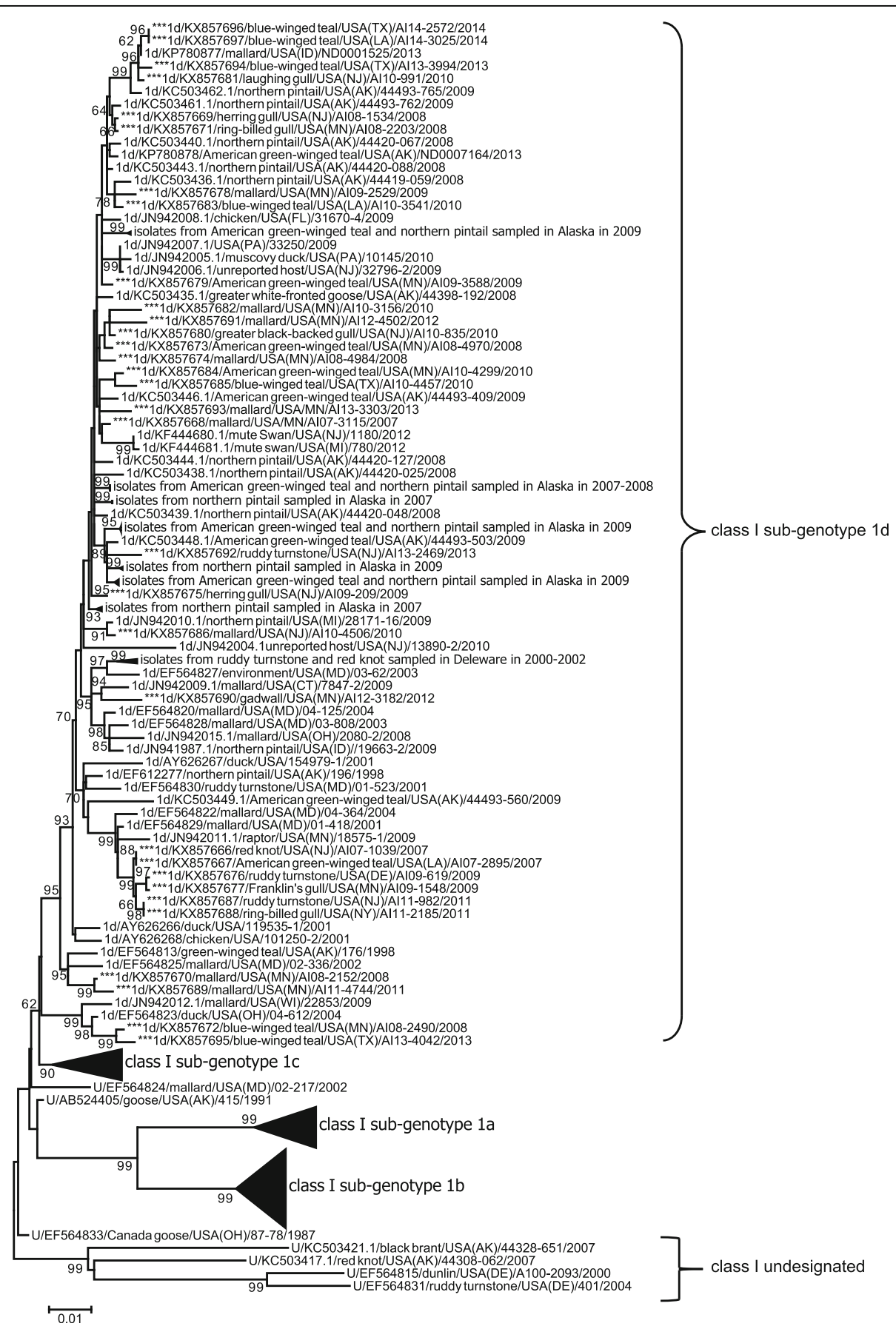

Fig. 1 Condensed maximum likelihood phylogenetic tree depicting inferred relationship among complete nucleotide sequences for the fusion protein gene of APMV-1 class I isolates. Bootstrap support values $\geq 60$ are shown to the left of branches. The tree is drawn to scale, with branch lengths measured in the number of substitutions per site. Tip labels for sequences are in the following format: taxonomic classification of isolate by sub-genotype $(U=$ unclassified)/ GenBank accession number/host/country of sample origin (and abbreviation for U.S. state if applicable)/isolate id/year of sample collection. Sequences generated for this study are indicated with asterisks $\left({ }^{* *}\right)$. The complete expanded phylogeny is provided as Additional file 2: Figure S1 
Table 2 Average nucleotide distance between sub-genotypes for class I APMV-1 isolates

\begin{tabular}{llll}
\hline Sub-genotype & $1 \mathrm{~b}$ & $1 \mathrm{c}$ & $1 \mathrm{~d}$ \\
\hline $1 \mathrm{a}$ & 0.074 & 0.076 & 0.079 \\
$1 \mathrm{~b}$ & & 0.078 & 0.077 \\
1c & & & 0.037 \\
\hline
\end{tabular}

generated for this study revealed less than 0.100 average genetic differences per site among isolates, consistent with designation as a single genotype (Fig. 1; Additional file 2: Figure S1). However, four clades of sequences within class I had an average distance per site between 0.037 and 0.079 (Table 2) and bootstrap support $\geq 60$ (Fig. 1; Additional file 2: Figure S1) supporting the designation of four sub-genotypes, $1 \mathrm{a}-1 \mathrm{~d}$, as per the nomenclature criteria for APMV-1 put forth by Diel et al. [33].

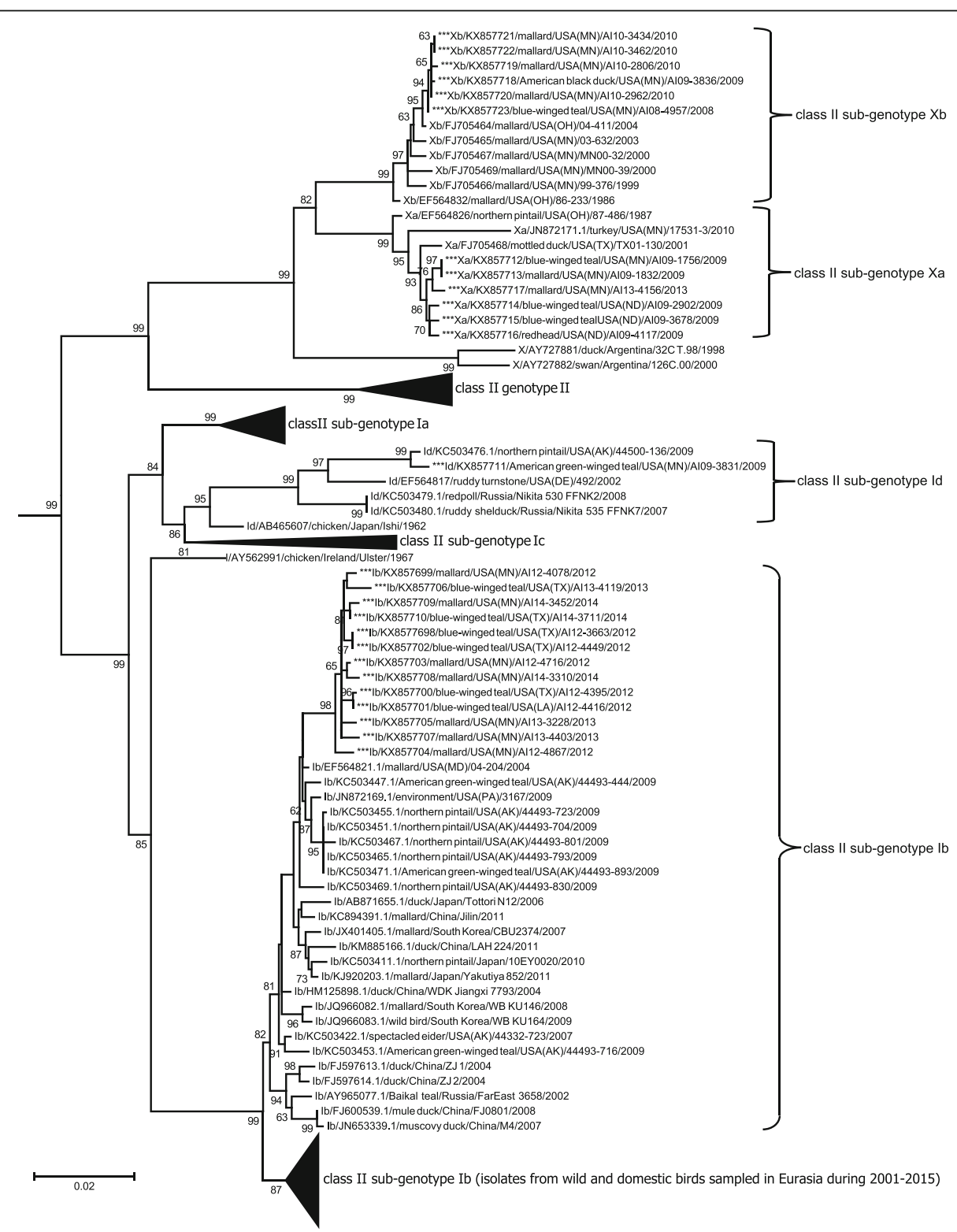

Fig. 2 Partial condensed maximum likelihood phylogenetic tree depicting inferred relationship among complete nucleotide sequences for the fusion protein gene of APMV-1 class II genotype I, II, and X isolates. Bootstrap support values $\geq 60$ are shown to the left of branches. The tree is drawn to scale, with branch lengths measured in the number of substitutions per site. Tip labels for sequences are in the following format: taxonomic classification of isolate by sub-genotype/GenBank accession number/host/country of sample origin (and abbreviation for U.S. state if applicable)/isolate id/year of sample collection. Sequences generated for this study are indicated with asterisks ${ }^{* * *}$ ). The complete expanded phylogeny is provided as Additional file 2: Figure $\mathbf{S 2}$ 
All 32 class I isolates sequenced for this study clustered within the clade classified as sub-genotype 1d (Fig. 1; Additional file 2: Figure S1). None of these 32 isolates had deduced amino acid motifs for the fusion protein cleavage site predictive of high virulence in poultry (data not shown).

Previously reported APMV-1 class II isolates and 26 additional closely-related sequences generated for this study clustered into seventeen clades meeting criteria for designation as distinct genotypes in ML phylogenetic analyses including an average of $>0.100$ differences per site [33]. Sequences previously designated as genotype $\mathrm{XV}$ appeared to represent only recombinant viruses [25] and were thus excluded from the current study. Fourteen isolates sequenced for this study clustered within the clade classified as genotype I and 12 isolates sequenced for this investigation grouped with sequences in genotype X (Fig. 2; Additional file 2: Figure S2). None of these isolates clustering within genotype I or genotype $\mathrm{X}$ clades had deduced amino acid motifs for the fusion protein cleavage site predictive of high virulence in poultry (data not shown). Within class II genotype I, sequences clustered into four sub-clades with an average distance per site between 0.061 and 0.069 supporting the designation of four sub-genotypes (Ia-Id; Table 3). For class II genotype $\mathrm{X}$, sequences clustered into two clades with an average distance per site of 0.047 supporting the designation of two sub-genotypes, $\mathrm{Xa}$ and $\mathrm{Xb}$ (Table 3).

\section{Assessment of inter-taxa/inter-region exchange}

When we considered a sub-set of 180 fusion gene sequences originating from wild birds samples throughout North America, the ML phylogenetic tree (Additional file 2: Figure S3) depicted inferred genetic relationships consistent with ML trees to assess contemporary genetic diversity (Figs. 1 and 2) and Bayesian phylogenetic analysis used to assess associations between branch tip traits and tree topology (Figs. 3, 4, 5, 6 and 7). Fusion gene sequences for 180 wild bird origin isolates clustered into four well-supported clades (Fig. 3). These four clades corresponded to: 1 . class I sub-genotype $1 \mathrm{~d}$ and six undesignated viral sequences, 2. class II genotype I (including sub-genotypes Ib-Id), 3. class II genotype V (sub-genotype $\mathrm{Va}$ ), and 4. class II genotype X (sub-genotypes $\mathrm{Xa}-\mathrm{Xb}$;

Table 3 Average nucleotide distance between sub-genotypes for class I| genotype I and class || genotype X APMV-1 isolates

\begin{tabular}{llllll}
\hline Sub-genotype & Ib & Ic & Id & Xa & Xb \\
\hline la & 0.069 & 0.061 & 0.062 & 0.103 & 0.102 \\
Ib & & 0.080 & 0.075 & 0.113 & 0.112 \\
Ic & & 0.070 & 0.119 & 0.117 \\
Id & & & 0.114 & 0.114 \\
Xa & & & & & 0.047 \\
\hline
\end{tabular}

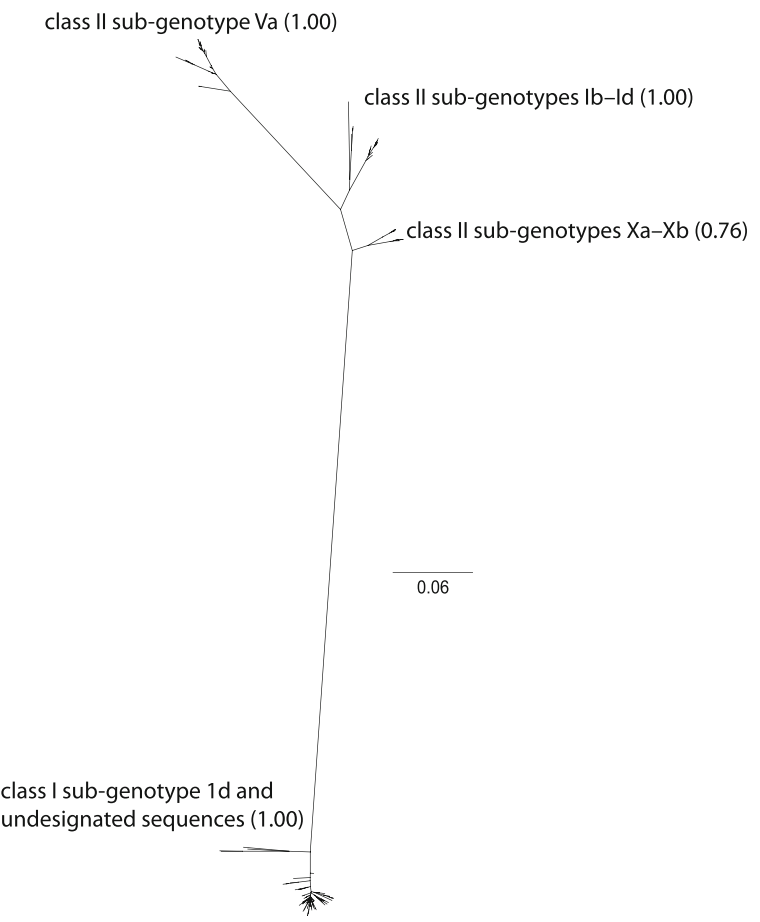

Fig. 3 Radial Bayesian phylogenetic tree depicting the inferred genetic relationship among fusion gene sequences for 180 APMV-1 isolates originating from samples collected from wild birds in North America. Posterior probability values for major clades are shown. Dendrograms for each major clade with complete tip labels and classification of individual sequences by taxonomic order of host and North American region of sample origin are provided as Figs. 4, 5, 6 and 7

Fig. 3). The clade comprised of sequences designated as class I sub-genotype 1d, in addition to six undesignated viral sequences, included isolates originating from waterfowl (Anseriformes) and gull/shorebird (Charadriiformes) samples collected at five of six geographic regions designated in this study (Fig. 4). Canada, the region to which the fewest APMV-1 fusion gene sequences from wild birds were assigned in this study $(n=3)$, was the only geographic location unrepresented in this clade.

The clade consisting of class II genotype I APMV-1 fusion gene sequences included viruses isolated from waterfowl (Anseriformes) and shorebird (Charadriiformes) samples originating from four geographic regions: Alaska, the Midwest, the Gulf Coast, and the East (Fig. 5). However, sequences assigned to genotype I sub-genotype Ib were exclusively from Anseriformes hosts (Fig. 5). In contrast, the clade consisting of sequences assigned to class II sub-genotype $\mathrm{Va}$ had differences with regard to both host and geographic region of origin. Fusion gene sequences in this clade were represented by viruses isolated primarily from cormorants (Suliformes; $n=38$ ) with few sequences also originating from gulls (Charadriiformes; $n=2$ ) and a pelican (Pelecaniformes; $n=1$; Fig. 6). APMV-1 isolates clustering in this clade originated from samples collected 


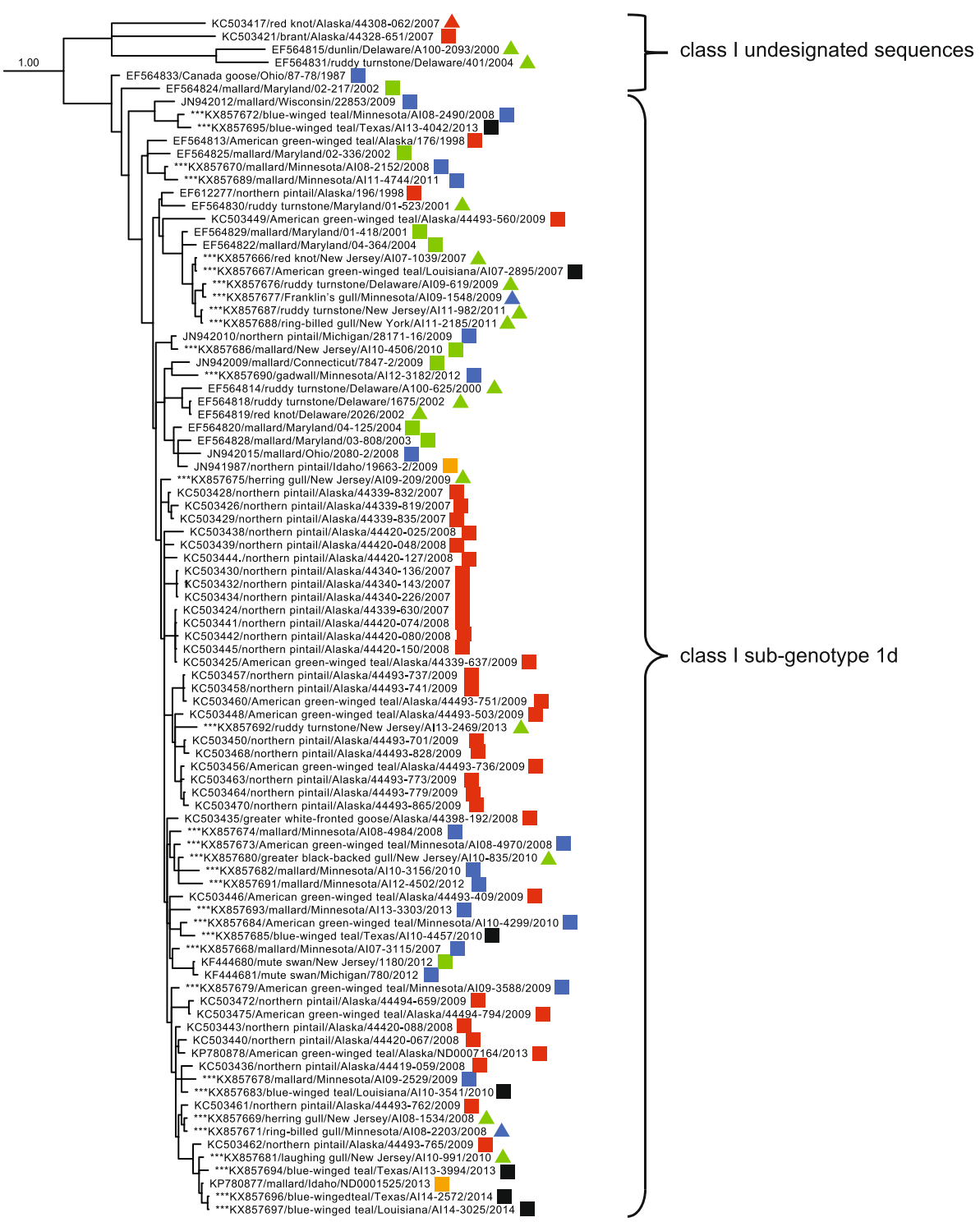

Fig. 4 Partial Bayesian phylogenetic tree depicting the inferred genetic relationship among fusion gene sequences for 92 APMV-1 isolates originating from samples collected from wild birds in North America and defined as class I sub-genotype $1 \mathrm{~d}$ or undesignated within class I. The Bayesian posterior probability for this major clade is indicated to the left of the first node. Sequences generated for this study are indicated with asterisks (***). Taxonomic order of host (Anseriformes = square, Charadriiformes $=$ triangle) and region of sample collection (Alaska $=$ red, West $=$ orange, Midwest $=$ blue, Gulf Coast $=$ black, East $=$ green) are indicated

at five of six North American regions identified in this study with a lack of sequences from Alaska (Fig. 6). Finally, the clade consisting of APMV-1 fusion gene sequences assigned to genotype $\mathrm{X}$ included viruses isolated exclusively from waterfowl (Anseriformes; Fig. 7). Nineteen of twenty sequences included in this clade originated from samples collected in the Midwest (Fig. 7).

When we investigated phylogenetic signals indicating limited viral transmission among divergent host taxa, trait association tests provided support for restricted APMV-1 exchange among hosts of different taxonomic orders. The
AI and PS for our phylogeny both provided support $(p<$ $0.01)$ for associations among branch tips when considering host taxa (Table 4). Furthermore, the MC provided evidence for associations between host taxonomic order for branch tips and tree topology for Anseriformes, Charadriiformes, and Suliformes ( $p \leq 0.01$; Table 4$)$. Statistically significant associations with regard to host order were driven by monophyletic clades of APMV-1 sequences in class I sub-genotype 1d (Charadriiformes), class II sub-genotype Ib (Anseriformes), class II sub-genotype Va (Suliformes), and class II genotype X (Anseriformes). 


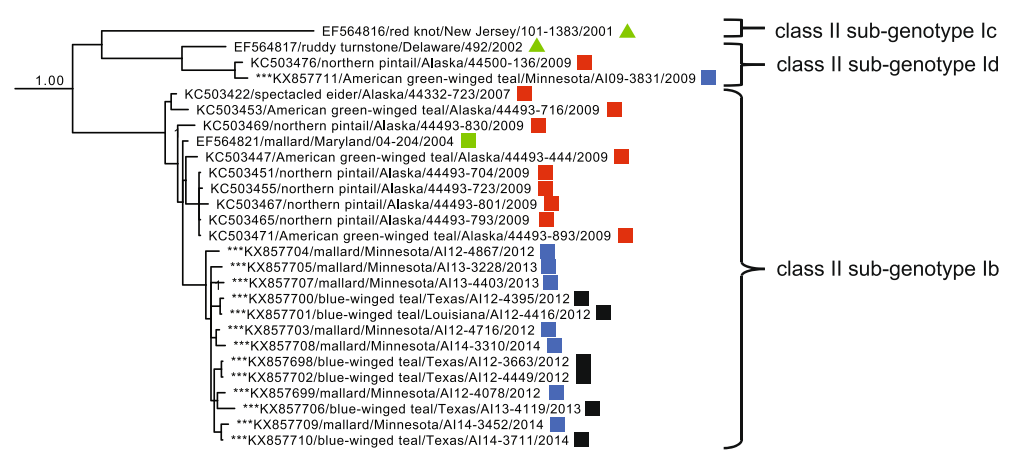

Fig. 5 Partial Bayesian phylogenetic tree depicting the inferred genetic relationship among fusion gene sequences for 27 APMV-1 isolates originating from samples collected from wild birds in North America and defined as class II sub-genotypes Ib-ld. The Bayesian posterior probability for this major clade is indicated to the left of the first node. Sequences generated for this study are indicated with asterisks (**). Taxonomic order of host (Anseriformes $=$ square, Charadriiformes $=$ triangle) and region of sample collection (Alaska $=$ red, Midwest $=$ blue, Gulf Coast $=$ black, East $=$ green $)$ are indicated

Using a similar approach to assess relationships between the tip trait of geographic region of sample origin and Bayesian phylogenetic tree topology, we also found statistical support for restricted geographic exchange of APMV-1 within North America. Both the AI and PS metrics provided support for associations between region of sample origin for branch tips and the topology of our phylogeny $(p<0.01$; Table 5$)$. Additionally, MC scores provided evidence for associations between tree topology and branch tips for the geographic regions of Alaska, the West, and the Midwest $(p \leq 0.01$; Table 5). These statistically significant associations with regard to region of sample origin were driven by monophyletic clades of APMV-1 sequences within class I sub-genotype 1d (Alaska), class II sub-genotype Ib (Alaska), class II sub-genotype Va (the West), and class II genotype X (the Midwest).

\section{Discussion}

In this study, we found evidence to support the designation of several previously undescribed APMV-1 subgenotypes, specifically class I sub-genotype $1 \mathrm{~d}$, class II sub-genotype Id, and class II sub-genotypes $\mathrm{Xa}$ and $\mathrm{Xb}$, based on our global phylogeny of fusion gene sequences

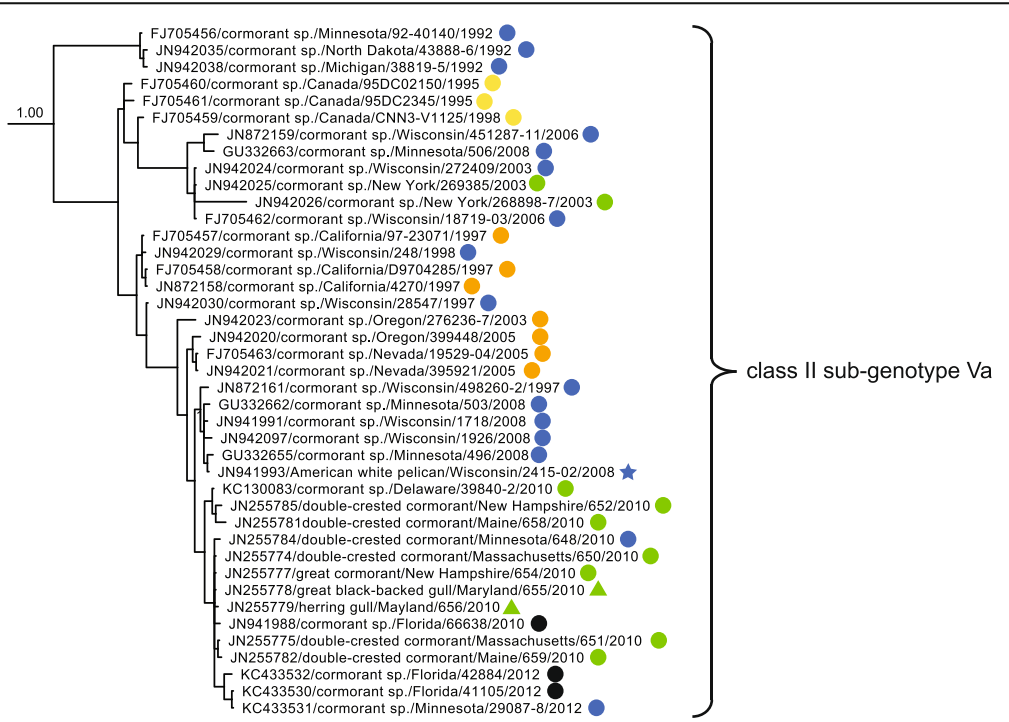

Fig. 6 Partial Bayesian phylogenetic tree depicting the inferred genetic relationship among fusion gene sequences for 41 APMV-1 isolates originating from samples collected from wild birds in North America and defined as class II sub-genotype Va. The Bayesian posterior probability for this major clade is indicated to the left of the first node. Sequences generated for this study are indicated with asterisks (***). Taxonomic order of host $($ Charadriiformes $=$ triangle, Suliformes $=$ circle, Pelecaniformes $=$ star) and region of sample collection $($ Canada $=$ yellow, West $=$ orange, Midwest $=$ blue, Gulf Coast $=$ black, East $=$ green $)$ are indicated 


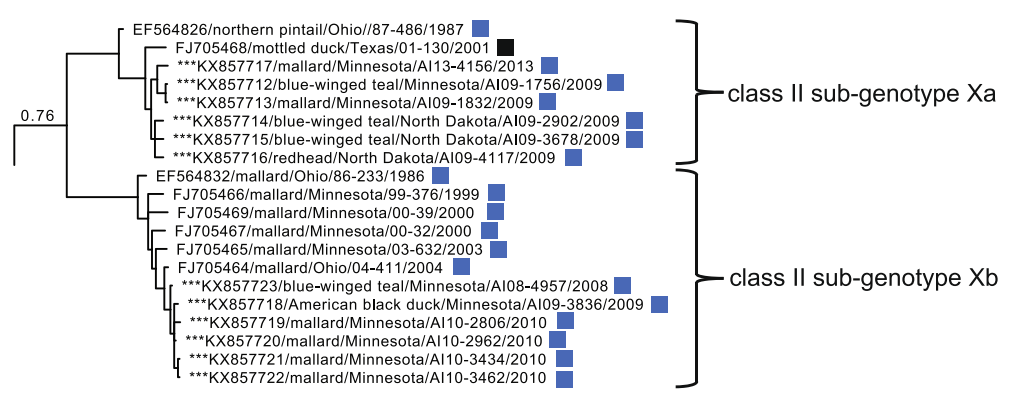

Fig. 7 Partial Bayesian phylogenetic tree depicting the inferred genetic relationship among fusion gene sequences for 20 APMV-1 isolates originating from samples collected from wild birds in North America and defined as class II sub-genotypes Xa-Xb. The Bayesian posterior probability for this major clade is indicated to the left of the first node. Sequences generated for this study are indicated with asterisks (***). Taxonomic order of host (Anseriformes $=$ square) and region of sample collection (Midwest $=$ blue, Gulf Coast $=$ black) are indicated

which included 58 previously unpublished wild bird origin isolates. This finding provides evidence that the full diversity of APMV-1 in wild birds has not previously been recognized and/or that this viral agent continues to evolve in natural reservoir hosts. The lack of identification of fusion gene sequences predictive of high pathogenicity in poultry from 58 previously uncharacterized wild bird APMV-1 isolates provides additional support for little or no role of the species sampled in this study (i.e., ducks, gulls, and shorebirds) in the emergence or maintenance of virulent strains of APMV-1 in North America [10, 11, 13, 39].

Inferred phylogenetic relationships among fusion gene sequences originating from wild bird samples provides evidence for exchange of APMV-1 among birds of different taxonomic orders and among geographic regions of North America; however, significant associations between tree topology and tip traits as identified through BaTS analyses also suggests that viral exchange may be greater among taxa of the same taxonomic order or within particular regions. For example, regarding the exchange of APMV-1 among taxa, entire clades of fusion gene sequences for class II sub-genotype Ib and class II genotype $\mathrm{X}$ were comprised exclusively of waterfowl (Anseriformes) isolates and had monophyletic clade sizes significantly larger than 95\% confidence intervals for what would be expected by chance. This suggests that this viral diversity may have evolved and been maintained exclusively in waterfowl species. Alternatively, unidentified sampling and diagnostic biases may have led to this result. We acknowledge that the species represented in this study were limited and may not reflect the full host range of APMV-1 lineages.

In contrast to the finding of monophyletic clades of sequences from Anseriformes hosts, the large clade comprised of class I sub-genotype 1d sequences contained numerous isolates originating from both waterfowl and gulls/shorebirds (Charadriiformes) with smaller monophyletic sub-clades of waterfowl sequences. Thus, there is evidence to suggest that class I sub-genotype $1 \mathrm{~d}$ APMV-1 may be readily shared among Anseriformes and Charadriiformes hosts. There were, however, two small monophyletic sub-clades of Charadriiformes sequences within the greater class I sub-genotype 1d clade which were significantly larger than expected by chance. As these gull/shorebird isolates were derived from samples collected in different regions and/or years, it is plausible that viral diversity is occasionally maintained exclusively within Charadriiformes hosts and then infrequently exchanged with sympatric waterfowl hosts. Additional and more systematic sampling for APMV-1 in Anseriformes and Charadriiformes hosts would be

Table 4 Bayesian tip-association significance testing results for APMV-1 phylogeny for wild birds isolates by host order

\begin{tabular}{lccccccc}
\hline Statistic & Observed mean & Lower 95\% CL & Upper 95\% CL & Null mean & Lower 95\% CL & Upper 95\% CL & Significance \\
\hline Al & 1.80 & 1.31 & 2.30 & 9.20 & 7.92 & 10.32 & $0.00^{*}$ \\
PS & 16.46 & 16.00 & 17.00 & 55.92 & 52.60 & 58.93 & $0.00^{*}$ \\
MC (Anseriformes) & 23.00 & 23.00 & 23.00 & 6.56 & 4.26 & 9.65 & $0.01^{*}$ \\
MC (Suliformes) & 8.77 & 7.00 & 9.00 & 2.24 & 1.51 & 3.01 & $0.01^{*}$ \\
MC (Charadriiformes) & 3.93 & 3.00 & 4.00 & 1.57 & 1.00 & 2.08 & $0.01^{*}$ \\
MC (Pelecaniformes) & 1.00 & 1.00 & 1.00 & 1.00 & 1.00 & 1.00 & 1.00 \\
\hline
\end{tabular}

Definitions of abbreviations are: $\mathrm{Al}=$ Association Index, $\mathrm{PS}=$ Fitch parsimony score, $\mathrm{MC}=$ monophyletic clade size statistic, and $\mathrm{CL}=$ confidence limit. Asterisks indicate results considered to be statistically significant $(p \leq 0.05)$ 
Table 5 Bayesian tip-association significance testing results for APMV-1 phylogeny for wild birds isolates by geographic region of sample origin

\begin{tabular}{|c|c|c|c|c|c|c|c|}
\hline Statistic & Observed mean & Lower 95\% CL & Upper 95\% CL & Null mean & Lower 95\% CL & Upper 95\% CL & Significance \\
\hline $\mathrm{Al}$ & 6.72 & 6.10 & 7.35 & 13.94 & 12.71 & 15.17 & $0.00^{*}$ \\
\hline PS & 54.69 & 53.00 & 57.00 & 96.43 & 92.06 & 101.92 & $0.00^{*}$ \\
\hline MC (Alaska) & 7.03 & 6.00 & 10.00 & 2.55 & 1.97 & 3.51 & $0.01^{*}$ \\
\hline MC (Canada) & 1.31 & 1.00 & 2.00 & 1.00 & 1.00 & 1.00 & 1.00 \\
\hline MC (West) & 3.00 & 3.00 & 3.00 & 1.10 & 1.00 & 1.99 & $0.01^{*}$ \\
\hline MC (Midwest) & 10.91 & 11.00 & 11.00 & 3.05 & 2.09 & 4.26 & $0.01^{*}$ \\
\hline MC (Gulf Coast) & 2.05 & 2.00 & 2.00 & 1.34 & 1.00 & 2.00 & 0.14 \\
\hline MC (East) & 3.21 & 3.00 & 5.00 & 2.10 & 1.47 & 3.01 & 0.12 \\
\hline
\end{tabular}

Definitions of abbreviations are: $\mathrm{Al}=$ Association Index, $\mathrm{PS}=$ Fitch parsimony score, $\mathrm{MC}=$ monophyletic clade size statistic, and $\mathrm{CL}=\mathrm{confidence} \mathrm{limit}$. Asterisks indicate results considered to be statistically significant $(p<0.05)$

beneficial for improving inference on the viral dynamics between these taxonomic groups.

Similar to evidence for restricted exchange of APMV-1 observed for a sub-set of the viral diversity detected in waterfowl, the clade of sequences designated as class II sub-genotype Va was comprised nearly exclusively of sequences from cormorants (Suliformes) with the exception of two sequences originating from gulls (Charadriiformes) and a single viral sequence from a pelican (Pelecaniformes). As such, the mean monophyletic clade of cormorant APMV-1 fusion gene sequences within the class II subgenotype Va clade exceeded the upper $95 \%$ confident limit expected by chance and provides support the for the evolution and maintenance of such viruses by cormorants. Other infected hosts, such as gulls and pelicans, are likely the result of spill-over infections. We caution, however, that as stated previously for other APMV-1 lineages, the possibility that unidentified sampling and diagnostic biases affected our results cannot be dismissed.

With regard to the exchange of APMV-1 among regions, clades for all four genotypes in our phylogeny for North American wild bird isolates were comprised of sequences originating from more than one region which suggests some degree of geographic dispersal of this viral agent by migratory birds. However, significant associations of branch tips for three regions within our phylogeny also provides evidence for either epidemiologic linkage of viruses analyzed in this study (e.g., viruses related to a single outbreak) or unrecognized mechanisms in hosts or the environment that have resulted in restrictions in the geographic extent of viral exchange for sub-sets of the genetic diversity of APMV-1. For example, statistical support for associations between region of sample origin and tree topology was observed for Alaska and the West. These results were driven by monophyletic clades of APMV-1 sequences for viruses isolated from waterfowl (Anseriformes) in Alaska (class I sub-genotype $1 \mathrm{~d}$ and class II sub-genotype Ib) and cormorants (Suliformes) in the West (genotype Va). All of these monophyletic clades represent sequences derived from isolates recovered from samples originating within the same region within a span of three years. Thus, statistical support for associations of tip traits within these clades may be best explained by exchange of viruses among sympatric taxa or infection from a common source during localized outbreaks rather than biotic or abiotic limits to viral dispersal into or out of Alaska and the West.

Stronger evidence for biologically significant geographic associations of branch tip traits and tree topology for APMV-1 was evidenced by the genetic relationship among sequences designated as class II genotype X. Nineteen of 20 sequences assigned to this genotype originated from waterfowl in the Midwest with the single exception being an isolate recovered from a mottled duck (Anas fulvigula) in Texas. As genotype $\mathrm{X}$ isolates sequenced for this study were recovered from samples collected from 1986-2013, a temporal period spanning 27 years, it is unlikely epidemiologic linkage related to a single outbreak can completely explain this result. Although genotype $\mathrm{X}$ isolates have also previously been detected in waterfowl in Argentina, isolates from North America are phylogenetically distinct from those detected in South America (Fig. 2; Additional file 2: Figure S2). Thus, there may be biotic or abiotic limitations restricting the circulation of APMV-1 class II genotype $\mathrm{Xa}-\mathrm{Xb}$ viruses beyond waterfowl inhabiting the Midwest that warrant additional investigation. Alternatively there may be cryptic epidemiological influences in our sampling approach that influenced our results (e.g., peak in prevalence of genotype $\mathrm{X}$ viruses coincident with sampling events in the Midwest or an unsampled reservoir host for such viruses with restricted range).

\section{Conclusion}

In summary, through analysis of APMV-1 fusion gene sequences for isolates recovered from wild birds sampled in North America, we found evidence for previously unrecognized viral diversity circulating among ducks, 
gulls, and shorebirds. We did not, however, find any evidence that such diversity was likely to be pathogenic to poultry or that this diversity may contribute to the emergence of avian pathogens in North America. When we assessed the exchange of APMV-1 among taxa, we found evidence for incomplete restrictions in viral sharing among taxa for some viral lineages (e.g., class II subgenotypes $\mathrm{Ib}, \mathrm{Va}, \mathrm{Xa}$, and $\mathrm{Xb}$ ) whereas other viral diversity appeared to be more readily exchanged among taxa (e.g., class I sub-genotype 1d). Finally, while our results support incomplete restrictions with regard to geographic dispersal by migratory birds for some APMV-1 viral lineages, this result was likely influenced by epidemiologic linkage. However, the association between class II genotype $\mathrm{X}$ viruses and waterfowl in the Midwest warrants further investigation.

\section{Additional files}

Additional file 1: Table S1. APMV-1 complete fusion protein gene sequences used for constructing maximum likelihood phylogenetic tree for assessment of genetic diversity. Sequences generated as part of this study are indicated in bold font. (XLSX $89 \mathrm{~kb}$ )

Additional file 2: Figure S1. Maximum Likelihood phylogeny depicting the inferred relationship among fusion gene sequences for APMV-1 class I isolates available on The National Center for Biotechnology Information GenBank as of 26 August $2016(n=211)$. The tree with the highest log likelihood (-14761.8677) is shown. Bootstrap support values $\geq 60$ are shown. The tree is drawn to scale, with branch lengths depicting the number of substitutions per site. All positions containing gaps and missing data were eliminated. There were a total of 1662 positions in the final dataset. Branch tips include sub-genotypes assigned to major clades meeting criteria described by Diel et al. (2012; $U$ = unclassified), followed by the GenBank accession number, host name, country of isolation, strain designation and year of isolation (if available). Sequences generated for this study are indicated with asterisks ${ }^{(* *)}$. Figure $\mathbf{S 2}$. Maximum Likelihood phylogeny depicting the inferred relationship among fusion gene sequences for APMV-1 class II isolates available on The National Center for Biotechnology Information GenBank as of 26 August 2016 ( $n=1272)$. The tree with the highest log likelihood (-82565.4161) is shown. Bootstrap support values > 60 are shown. The tree is drawn to scale, with branch lengths depicting the number of substitutions per site. All positions containing gaps and missing data were eliminated. There were a total of 1644 positions in the final dataset. Branch tips include sub-genotypes assigned to major clades meeting criteria described by Diel et al. (2012; U = unclassified), followed by the GenBank accession number, host name, country of isolation, strain designation and year of isolation (if available). Sequences generated for this study are indicated with asterisks $\left(^{* * *}\right)$. Figure S3. Maximum Likelihood phylogenetic tree depicting the inferred genetic relationship among fusion gene sequences for 180 APMV-1 isolates originating from samples collected from wild birds in North America. Bootstrap support values for major clades are shown. Sequences generated for this study are indicated with asterisks $(* * *)$. Taxonomic order of host (Anseriformes $=$ square, Charadriiformes $=$ triangle, Suliformes $=$ circle, Pelecaniformes $=$ star) and region of sample collection (Alaska $=$ red, Canada $=$ yellow, West $=$ orange, Midwest $=$ blue, Gulf Coast $=$ black, East $=$ green $)$ are indicated. (PDF $1177 \mathrm{~kb})$

\section{Abbreviations}

Al: Association Index; APMV-1: Avian paramyxovirus serotype 1; MC: Monophyletic clade size statistic; PS: Fitch parsimony score; RNA: Ribonucleic acid; USA: United States of America

\section{Acknowledgements}

We appreciate field and laboratory support by Deborah Carter, Nick Davis-Fields, Alinde Fojtik, Clara Kienzle, Paul Oesterle, Jeremiah Slagter, Dawn Williams-Coplin, and Ben Wilcox. We appreciate reviews provided by John Pearce, Andy Reeves, and two anonymous reviewers. Any use of trade, firm, or product names is for descriptive purposes only and does not imply endorsement by the U.S. Government. The USDA is an equal opportunity provider and employer.

\section{Funding}

This project was funded, in part, by the U.S. Department of Agriculture Agricultural Research Service Current Research Information System (604032000-072) and by the U.S. Geological Survey through the Wildlife Program of the Ecosystems Mission area.

\section{Availability of data and materials}

Genetic data presented in this paper are publicly available via GenBank and through the U.S. Geological Survey at https://dx.doi.org/10.5066/F7MP51GP.

\section{Authors' contributions}

AMR, KMD, and CLA conceived this project. DES coordinated field sampling efforts. RLP conducted viral screening. IVG sequenced viral isolates. AMR, JTH, KMD, and JB conducted genetic analyses and interpreted results. AMR, IVG, JTH, $K M D, R L P, D E S, J B$, and CLA wrote, revised, and/or reviewed the manuscript. All authors read and approved the final manuscript.

\section{Competing interests}

The authors declare that they have no competing interests.

\section{Consent for publication}

Not applicable.

\section{Ethics approval and consent to participate}

All procedures involving sampling of wild birds were approved by the University of Georgia Institutional Animal Care and Use Committee and collected under state and federal permits (Minnesota Division of Fish and Wildlife permit 16282, Federal Bird Banding Permits \#09072 and \#23792, U.S. Fish and Wildlife Service permits MB779283-0 and MB779238-2).

\section{Author details}

'US Geological Survey, Alaska Science Center, 4210 University Drive, Anchorage, Alaska 99508, USA. ${ }^{2}$ US Department of Agriculture, Agriculture Research Service, US National Poultry Research Center, Southeast Poultry Research Laboratory, 934 College Station Road, Athens, GA 30605, USA. ${ }^{3}$ University of Texas, School of Public Health, 1200 Pressler Street, Houston, TX 77030, USA. ${ }^{4}$ Department of Population Health, Southeastern Cooperative Wildlife Disease Study, College of Veterinary Medicine, University of Georgia, Athens, GA 30602, USA.

Received: 5 January 2017 Accepted: 17 February 2017

Published online: 03 March 2017

\section{References}

1. Wobeser G, Leighton FA, Norman R, Myers DJ, Onderka D, Pybus MJ, Neufeld JL, Fox GA, Alexander DJ. Newcastle disease in wild water birds in western Canada, 1990. Can Vet J. 1993;34:353-9.

2. Banerjee M, Reed WM, Fitzgerald SD, Panigrahy B. Neurotropic velogenic Newcastle disease in cormorants in Michigan: pathology and virus characterization. Avian Dis. 1994;38:873-8.

3. Kuiken T, Leighton FA, Wobeser G, Danesik KL, Riva J, Heckert RA. An epidemic of Newcastle disease in double-crested cormorants from Saskatchewan. J Wildl Dis. 1998;34:457-71.

4. Glaser LC, Barker IK, Weseloh DV, Ludwig J, Windingstad RM, Key DW, Bollinger TK. The 1992 epizootic of Newcastle disease in double-crested cormorants in North America. J Wildl Dis. 1999;35:319-30.

5. Diel DG, Miller PJ, Wolf PC, Mickley RM, Musante AR, Emanueli DC, Shively KJ, Pedersen K, Afonso CL. Characterization of Newcastle disease viruses isolated from cormorant and gull species in the United States in 2010. Avian Dis. 2012;56:128-33.

6. Panigrahy B, Senne DA, Pearson JE, Mixson MA, Cassidy DR. Occurrence of velogenic viscerotropic Newcastle disease in pet and exotic birds in 1991. Avian Dis. 1993;37:254-8. 
7. Clavijo A, Robinson Y, Booth T, Munroe F. Velogenic Newcastle disease in imported caged birds. Can Vet J. 2000;41:404-6.

8. Munir M, Shabbir MZ, Yaqub T, Shabbir MA, Mukhtar N, Khan MR, Berg M. Complete genome sequence of a velogenic neurotropic avian paramyxovirus 1 isolated from peacocks (Pavo cristatus) in a wildlife park in Pakistan. J Virol. 2012:86:13113-4.

9. Alexander DJ, Senne DA. Newcastle disease, other avian paramyxoviruses, and pneumovirus infections. In: Saif YM, Fadly AM, Glisson JR, McDougald LR, Nolan LK, Swayne DE, editors. Diseases of poultry. 12th ed. Ames: lowa State University Press; 2008. p. 75-100.

10. Zanetti F, Berinstein A, Pereda A, Taboga O, Carrillo E. Molecular characterization and phylogenetic analysis of Newcastle disease virus isolates from healthy wild birds. Avian Dis. 2005;49:546-50.

11. Kim LM, King DJ, Curry PE, Suarez DL, Swayne DE, Stallknecht DE, Slemons RD, Pedersen JC, Senne DA, Winker K, Afonso CL. Phylogenetic diversity among low-virulence Newcastle disease viruses from waterfowl and shorebirds and comparison of genotype distributions to those of poultryorigin isolates. J Virol. 2007;81:12641-53.

12. Kim LM, King DJ, Guzman H, Tesh RB, da Rosa Ap T, Bueno Jr R, Dennett JA, Afonso CL. Biological and phylogenetic characterization of pigeon paramyxovirus serotype 1 circulating in wild North American pigeons and doves. J Clin Microbiol. 2008;46:3303-10.

13. Ramey AM, Reeves AB, Ogawa H, Ip H, Imai K, Bui VN, Yamaguchi E, Silko NY, Afonso CL. Genetic diversity and mutation of avian paramyxovirus serotype 1 (Newcastle disease virus) in wild birds and evidence for intercontinental spread. Arch Virol. 2013;158:2495-503.

14. Senne DA, Pearson JE, Miller LD, Gustafson GA. Virus isolations from pet birds submitted for importation into the United States. Avian Dis. 1983:27:731-44.

15. Cardenas Garcia S, Navarro Lopez R, Morales R, Olvera MA, Marquez MA, Merino $\mathrm{R}$, Miller PJ, Afonso CL. Molecular epidemiology of Newcastle disease in Mexico and the potential spillover of viruses from poultry into wild bird species. Appl Environ Microbiol. 2013;79:4985-92.

16. Marín MC, Villegas P, Bennett JD, Seal BS. Virus characterization and sequence of the fusion protein gene cleavage site of recent Newcastle disease virus field isolates from the southeastern United States and Puerto Rico. Avian Dis. 1996:40:382-90

17. Seal BS, Wise MG, Pedersen JC, Senne DA, Alvarez R, Scott MS, King DJ, Yu Q, Kapczynski DR. Genomic sequences of low-virulence avian paramyxovirus(Newcastle disease virus) isolates obtained from live-bird markets in North America not related to commonly utilized commercial vaccine strains. Vet Microbiol. 2005;106:7-16

18. Kim LM, King DJ, Suarez DL, Wong CW, Afonso CL. Characterization of class ! Newcastle disease virus isolates from Hong Kong live bird markets and detection using real-time reverse transcriptase-PCR. J Clin Microbiol. 2007:45:1310-4.

19. Fan S, Wang T, Gao X, Ying Y, Li X, Li Y, Li Y, Ma J, Sun H, Chu D, Xu Y, Yang S, Li Q, Gao Y, Xia X. Phylogenetic analysis of Newcastle disease viruses isolated from wild birds in the Poyang Lake region of China. J Vet Med Sci. 2015;9:1143-9.

20. Heckert RA, Collins MS, Manvell RJ, Strong I, Pearson JE, Alexander DJ. Comparison of Newcastle disease viruses isolated from cormorants in Canada and the USA in 1975, 1990 and 1992. Can J Vet Res. 1996;60:50-4.

21. Kim B-Y, Lee D-H, Kim M-S, Jang J-H, Lee Y-N, Park J-K, Yuk S-S, Lee J-B, Park S-Y, Choi I-S, Song C-S. Exchange of Newcastle disease viruses in Korea: The relatedness of isolates between wild birds, live bird markets, poultry farms and neighboring countries. Infect Genet Evol. 2012;12:478-82.

22. Snoeck CJ, Adeyanju AT, Owoade AA, Couacy-Hymann E, Alkali BR, Ottosson U, Muller CP. Genetic diversity of Newcastle disease virus in wild birds and pigeons in West Africa. Appl Environ Microbiol. 2013;79:7867-74.

23. Ayala AJ, Dimitrov KM, Becker CR, Goraichuk IV, Arns CW, Bolotin VI, Ferreira HL, Gerilovych AP, Goujgoulova GV, Martini MC, Muzyka DV, Orsi MA, Scagion GP, Silva RK, Solodiankin OS, Stegniy BT, Miller PJ, Afonso CL. Presence of vaccinederived Newcastle disease viruses in wild birds. PLoS One. 2016;11:e0162484

24. Gould AR, Kattenbelt JA, Selleck P, Hansson E, Della-Porta A, Westbury HA. Virulent Newcastle disease in Australia: molecular epidemiological analysis of viruses isolated prior to and during the outbreaks of 1998-2000. Virus Res. 2001;77:51-60.

25. Dimitrov KM, Ramey AM, Qiu X, Bahl J, Afonso CL. Temporal, geographic, and host distribution of avian paramyxovirus 1 (Newcastle disease virus). Infect Genet Evol. 2016;39:22-34.

26. Wilcox BR, Knutsen GA, Berdeen J, Goekjian V, Poulson R, Goyal S, Sreevatsan S, Cardona C, Berghaus RD, Swayne DE, Yabsley MJ, Stallknecht DE. Influenza-A viruses in ducks in northwestern Minnesota: fine scale spatial and temporal variation in prevalence and subtype diversity. PLoS One. 2011;6:e24010.

27. Stallknecht DE, Luttrel MP, Poulson R, Goekjian V, Niles L, Dey A, Krauss S, Webster RG. Detection of avian influenza viruses from shorebirds: evaluation of surveillance and testing approaches. J Wildl Dis. 2012;48:382-93.

28. Ramey AM, Poulson RL, González-Reiche AS, Wilcox BR, Walther P, Link P, Carter DL, Newsome GM, Müller ML, Berghaus RD, Perez DR, Hall JS, Stallknecht DE. Evidence for seasonal patterns in the relative abundance of avian influenza virus subtypes in blue-winged teal (Anas discors). J Wildl Dis. 2014;50:916-22.

29. Miller PJ, Dimitrov KM, Williams-Coplin D, Peterson MP, Pantin-Jackwood MJ, Swayne DE, Suarez DL, Afonso CL. International biological engagement programs facilitate Newcastle disease epidemiological studies. Front Public Health. 2015:3:235.

30. Benson DA, Clark K, Karsch-Mizrachi I, Lipman DJ, Ostell J, Sayers EW. GenBank. Nucleic Acids Res. 2015:43:D30-5.

31. Tamura K, Stecher G, Peterson D, Filipski A, Kumar S. MEGA6: molecular evolutionary genetics analysis version 6.0. Mol Biol Evol. 2013;30:2725-9.

32. Tamura K, Nei M, Kumar S. Prospects for inferring very large phylogenies by using the neighbor-joining method. Proc Natl Acad Sci U S A. 2004;101: 11030-5.

33. Diel DG, da Silva LHA, Liu H, Wang Z, Miller PJ, Afonso CL. Genetic diversity of avian paramyxovirus type 1: proposal for a unified nomenclature and classification system of Newcastle disease virus genotypes. Infect Genet Evol. 2012;12:1770-9.

34. Stamatakis A. RAxML version 8: a tool for phylogenetic analysis and postanalysis of large phylogenies. Bioinformatics. 2014;30:1312-3.

35. Ronquist F, Teslenko M, van der Mark P, Ayres DL, Darling A, Höhna S, Larget B, Liu L, Suchard MA, Huelsenbeck JP. MrBayes 3.2: efficient Bayesian phylogenetic inference and model choice across a large model space. Systematic Biol. 2012; 61:539-42.

36. Parker J, Rambaut AR, Pybus OG. Correlating viral phenotypes with phylogeny: accounting for phylogenetic uncertainty. Infect Genet Evol. 2008:8:239-46.

37. Wang TH, Donaldson YK, Brettle RP, Bell JE, Simmonds P. Identification of shared populations of human immunodeficiency virus type 1 infecting microglia and tissue macrophages outside the central nervous system. J Virol. 2001;75:11686-99.

38. Fitch WM. Toward defining the course of evolution: minimal change for a specific tree topology. Syst Zool. 1971;20:406-16.

39. Pedersen K, Marks DR, Afonso CL, Stopak SR, Williams-Coplin D, Dimitrov KM, Miller PJ, Deliberto TJ. Identification of avian paramyxovirus serotype-1 in wild birds in the USA. J Wildl Dis. 2016;52:657-62.

\section{Submit your next manuscript to BioMed Central and we will help you at every step:}

- We accept pre-submission inquiries

- Our selector tool helps you to find the most relevant journal

- We provide round the clock customer support

- Convenient online submission

- Thorough peer review

- Inclusion in PubMed and all major indexing services

- Maximum visibility for your research

Submit your manuscript at www.biomedcentral.com/submit
) Biomed Central 